Cinémas

Revue d'études cinématographiques

Journal of Film Studies

\title{
Le Spectacteur et le contactile
}

\section{Réjean Dumouchel}

Volume 1, numéro 3, printemps 1991

Nouvelles technologies : nouveaux cinémas?

URI : https://id.erudit.org/iderudit/1001065ar

DOI : https://doi.org/10.7202/1001065ar

Aller au sommaire du numéro

Éditeur(s)

Cinémas

ISSN

1181-6945 (imprimé)

1705-6500 (numérique)

Découvrir la revue

Citer cet article

Dumouchel, R. (1991). Le Spectacteur et le contactile. Cinémas, 1(3), 38-60. https://doi.org/10.7202/1001065ar

\section{Résumé de l'article}

Certains enjeux esthétiques fondamentaux d'une forme d'art issue d'un nouveau support technologique, l'oeuvre interactive, telle que rendue possible par la révolution micro-informatique et l'invention du disque laser, sont abordés ici: la présence d'un spectacteur (mot contractant spectateur et acteur), interrogé à la lumière des théories de Morin et de Metz, l'importance d'un contactile (mot contractant contact et tactile) et une conception nouvelle de l'image. d'utilisation que vous pouvez consulter en ligne.

https://apropos.erudit.org/fr/usagers/politique-dutilisation/ 


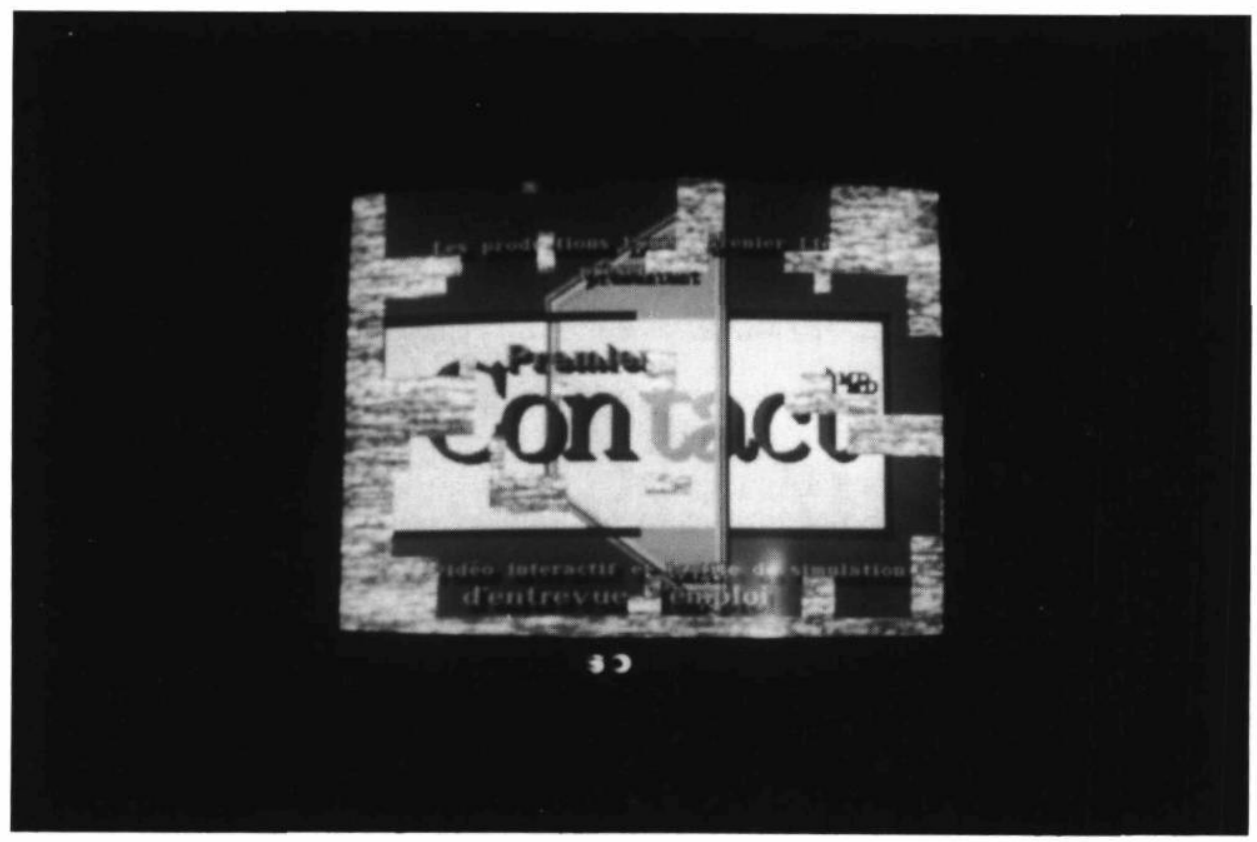

Premier contact de Réjean Dumouchel, Louis Fortier, Diane Grenier, Marie Grenier, Wilfrid Lamoureux. Les Productions Louise Grenier (1987)

Photo: Réjean Dumouchel 


\title{
Le Spectacteur et le contactile
}

\section{Réjean Dumouchel}

\begin{abstract}
RÉSUMÉ
Certains enjeux esthétiques fondamentaux d'une forme d'art issue d'un nouveau support technologique, l'œuvre interactive, telle que rendue possible par la révolution micro-informatique et l'invention du disque laser, sont abordés ici: la présence d'un spectacteur (mot contractant spectateur et acteur), interrogé à la lumière des théories de Morin et de Metz, l'importance d'un contactile (mot contractant contact et tactile) et une conception nouvelle de l'image.
\end{abstract}

\begin{abstract}
Interactive creative works are a new art form made possible by the new technological means created by the combination of microcomputers and laser disks. The role of the "spectactor" (spectator/actor) is examined from the standpoint of the theories of Morin and Metz. The importance of a "contactile" (contact/tactile) and a new concept of editing are discussed.
\end{abstract}

La poétique de l'œuvre en mouvement (et en partie aussi, celle de l'œuvre "ouverte») instaure un nouveau type de rapports entre l'artiste et son public, un nouveau fonctionnement de la perception esthétique; elle assure au produit artistique une place nouvelle dans la société. Elle établit enfin un rapport inédit entre la contemplation et l'utilisation de l'œuvre d'art (Éco, p. 37).

Cet extrait de l'ouvrage L'EEuvre ouverte d'Umberto Éco rejoint la volonté des artistes à remettre en question la passivité du spectateur et l'immobilité de l'œuvre. Une ouvre en mouvement (toute œuvre dont la forme change selon l'intervention du lecteur-exécutant) ou ouverte (dont l'ambiguïté permet une infinité d'interprétations conditionnées par la forme de l'œuvre) sont ce qu'aujourd'hui nous appelons une œuvre interactive, dans la mesure où cette œuvre offre prise à une certaine manipulation de la part du consommateur. 
Nous tenterons de dégager certains aspects de l'œuvre interactive. Par interactif, nous entendons la qualité d'un système à être manipulé en temps réel par l'usager, en ce qui a trait au déroulement de l'information qu'il diffuse. Cette qualité d'interactivité possède divers degrés qui s'échelonnent d'une interactivité minimale, alors que l'usager a peu d'initiative, jusqu'à un degré idéal, lorsque cette fois l'usager communique de façon libre avec un système dont les réactions suggèrent qu'il est en vie. Le niveau d'interactivité d'une œuvre se mesure à la liberté créative qu'elle partage avec le public. Cependant, il faut préciser que l'interactivité est vue différemment selon que l'on traite du processus de production d'une œuvre ou de celui de sa consommation. Dans le premier cas, l'interactivité est synonyme de souplesse de l'outil informatique qui permet à l'artiste-producteur une grande latitude dans la manipulation (par exemple les logiciels de dessin qui permettent la production d'images numériques) et beaucoup de puissance dans le calcul en temps réel des modifications apportées au dessin. Dans le deuxième cas, la notion d'interactivité sous-entend la possibilité de manipuler l'information de façon à ce qu'elle corresponde aux choix explicites du consommateur. Ce dernier se voit offrir une palette de possibilités d'intervention dans l'œuvre, ce qui influence le déroulement linéaire de celle-ci. Nous traiterons exclusivement de ce mode d'interactivité, rendu possible par l'alliance de l'informatique et du disque laser.

L'avènement de l'interactivité est en effet inséparable de la révolution micro-informatique et de l'invention du disque laser. Par la miniaturisation des composantes électroniques des lecteurs de vidéodisques d'une part et le développement des ordinateurs personnels d'autre part, de nouvelles possibilités voient le jour, accompagnées d'une souplesse d'utilisation sans précédent dans les médias audiovisuels. L'évolution, à la fois du matériel et du logiciel, recule sans cesse les limites expressives du vidéodisque en lui permettant de s'ajuster aux demandes ponctuelles de l'utilisateur. On assiste alors à la naissance d'un nouvel art: la fiction interactive.

Tandis que le cinéma comme l'architecture, la littérature, la poésie, la photographie et la bande dessinée sont des arts qui produisent, par essence, des objets donnés une fois pour toutes, éternellement pareils à eux-mêmes, réalité imaginée, inaltérable et figée, le disque laser en tant que support de la fiction interactive est un "art d'interprétation" comme la danse, le théâtre, la musique... Comme le jeu et le sport, la fiction interactive se joue, donc nécessite un ou des joueurs, tandis qu'un film, par 
exemple, joue, donc est lui-même joueur qui se donne en spectacle. Le cinéma, comme le théâtre ou la musique de concert, opère une séparation nette entre ceux et celles qui assistent au jeu et ceux et celles qui jouent dans l'enceinte circonscrite comme lieu de la représentation.

La fiction interactive est un spectacle où le public prend parti et assume une part du processus actif de la représentation. Le public participe et il serait tout aussi juste de parler de fiction participative. Le public acquiert un statut égal à celui du personnage et joue dans la fiction comme il joue avec la fiction, on peut alors parler de fiction ludique.

Dans une œuvre interactive, le spectateur quitte la salle et monte sur la scène. Il réclame le droit à la participation, l'opportunité de vivre dans le spectacle plutôt que de le regarder sans pouvoir d'intervention. Il réclame un nouveau statut: de spectateur, il devient acteur, finalement spectacteur.

\section{Le spectacteur ou le nouveau rôle du public}

Le mot spectacteur est un mot-valise contractant le mot spectateur et le mot acteur. Le spectacteur est un spectateur-acteur de l'œuvre interactive. Il est à la fois l'observateur du récit, celui pour qui le récit est dit ou montré, et le protagoniste du récit, celui par qui le récit se joue. C'est une double position assez délicate, il convient de la mettre en scène avec une certaine conscience du contexte nouveau dans lequel est placé le public qui n'est pas toujours rassuré par un tel type de communication.

Il est cependant tentant d'accorder au développement de la fiction interactive une fonction libératrice pour le public. Du spectateur passif au spectacteur impliqué dans l'exécution de l'œuvre, il y a bien «un nouveau type de rapport entre l'artiste et son public» (Éco, p. 37). Le public libéré des chaînes de la passivité n'est pourtant pas toujours au bout de ses peines et se voit quelquefois condamné aux travaux forcés.

Quand une œuvre n'a d'interactive que le nombre limité d'options qu'elle laisse au public, nous nous trouvons bien près du degré minimum d'interactivité. Il y va de tout le talent des concepteurs pour aménager des espaces et des temps dans la fiction où le public peut s'exprimer et personnaliser le récit. À ce titre, nous pouvons hasarder que la fiction interactive libère le public d'une passivité impuissante, et libère l'auteur de la contrainte de la rigidité diégétique.

L'auteur doit s'ouvrir au public autant que son œuvre peut le faire.

L'oeuvre en mouvement rend possible une multiplicité d'interventions personnelles, mais non pas de façon amorphe et 
vers n'importe quelle intervention. Elle est une invitation, non pas nécessitante ni univoque mais orientée, à une insertion relativement libre dans un monde qui reste celui voulu par l'auteur.

En somme, l'auteur offre à l'interprète une œuvre à achever. II ignore de quelle façon précise elle se réalisera, mais il sait qu'elle restera son œuvre; au terme du dialogue interprétatif, se concrétisera une forme organisée par un autre, mais une forme dont il reste l'auteur. Son rôle consiste à proposer des possibilités déjà rationnelles, orientées et dotées de certaines exigences organiques qui déterminent leur développement (Éco, p. 34).

Éco ne parle plus de public ou de spectateur mais d'interprète qui s'avère un des aspects du nouveau rôle que le public doit assumer face aux «œuvres en mouvement». Un interprète est le médiateur de l'œuvre d'art: un musicien interprète une partition, un comédien interprète un personnage, un spectacteur interprète une fiction interactive. Celle-ci s'interprète par le public qui la joue. Le public a un rôle et son rôle est en partie celui d'interprète, de médiateur de la fiction. Ce déplacement de rôle est important, par rapport au rôle passif donné au public par les masses-médias. En fait ce déplacement de rôle responsabilise le public face à l'œuvre représentée. Le public est responsable (en partie du moins) du déroulement de l'œuvre, de sa durée. Jusqu'à quel point peut-il en être responsable? Jusqu'à ce que le degré maximum d'interactivité soit atteint. Et ce n'est pas pour demain...

Quant à l'auteur, son rôle change aussi. Comme Éco le souligne, il s'agit dès lors de donner une œuvre structurellement complète mais formellement inachevée. Il faut tout prévoir et tout permettre. Le rôle de l'art en devient changé et un glissement vers le public s'effectue. Le piédestal de l'artiste tout puissant est ébranlé jusqu'à ce que de nouveaux artistes habités de cette dynamique se voient élevés par un public extrêmement intime avec l'œuvre. La relation entre l'artiste et son public en est alors une de performance. Chaque spectacteur performe dans l'œuvre et le plaisir retiré de cette performance se projette sur l'artiste qui reste le créateur d'un monde dans lequel le public s'adapte et agit.

Pour bien saisir ce nouveau rôle du public, sa transmutation en spectacteur, entreprenons une comparaison entre le processus psychologique mis en branle lors d'une représentation cinématographique, et celui qui est activé lors de l'actualisation d'une œuvre interactive par un spectacteur.

Plusieurs études ont été engagées sur le processus psychologique de la consommation d'un film. La plupart des auteurs ont comparé le spectacle cinématographique à un rêve que le ciné- 
phile a l'impression de faire. Tout comme la torpeur dans laquelle ce dernier est plongé lors de la projection rappelle le sommeil ou le demi-sommeil. Par exemple, Guy Corneau, dans son texte "Narcisse au cinéma», écrit: «Le film est un demisommeil, il a le pouvoir de nous délasser et il nous plonge dans une certaine solitude qui correspond à ce réinvestissement du soi et des soi-objets par la libido qui reflue» (p. 19).

Christian Metz renchérit:

(...) le sujet suspend ses investissements d'objets ou renonce du moins à leur frayer un débouché réel, et se replie pour un temps sur une base plus narcissique (plus introvertie dans la mesure où les fantasmes restent objectaux) (Metz, 1975, p.100).

Comme dans le sommeil, toute l'énergie psychique est intériorisée au cinéma, pêchant dans l'eau trouble du subconscient qui est le lieu de prise de connaissance des messages oniriques. Cependant, même si les auteurs parlent de sommeil dans le cas de l'état psychologique engendré par le spectacle cinématographique, ils se doivent de spécifier que l'état béat du spectateur au cinéma n'est pas le sommeil mais bien autre chose. Christian Metz prend soin de l'écrire: "La situation filmique porte en elle certains éléments d'une inhibition motrice, et elle est en cela un petit sommeil, un sommeil éveillé» (Metz, 1977, p. 142).

Plutôt paradoxale, cette expression de sommeil éveillé évoque le caractère ambigu de l'état psychologique du cinéphile. Les caractéristiques premières de cet état semblent l'inactivité du corps et le repliement sur soi. Ces deux caractéristiques rapprochent l'état de fascination dans lequel nous plonge le spectacle cinématographique à l'état de sommeil. Pas n'importe quel sommeil, mais bien celui peuplé de rêves. Encore là, comme dans le cas de sa comparaison avec le sommeil, l'état psycho-filmique, si l'on peut se permettre l'expression, n'est pas un état onirique, bien qu'il lui ressemble.

Christian Metz, dans le même ouvrage, pour remplacer le terme gênant de rêve, en suggère un autre, plus proche à son avis de l'état psycho-filmique:

En essayant de préciser les relations entre l'état filmique et l'état onirique, parentés partielles et divergentes, incomplètes, on rencontre à chaque pas le problème du sommeil, ou de son absence, ou de ses degrés intermédiaires. Aussi est-on inévitablement amené à introduire dans l'analyse un terme nouveau, la rêverie, qui, comme l'état filmique et contrairement au rêve, est une activité de la veille. Le Français, lorsqu'il veut distinguer plus nettement rêve de la rêverie, appelle cette dernière, d'un syntagme figé et redondant, «rêverie éveillée». C'est le Tagtraum de Freud, le rêve de jour, bref le fantasme conscient (Metz, 1977, p. 149). 
Metz introduit le terme de rêverie comme étant plus apte à décrire l'état filmique parce que dans la rêverie, nous sommes éveillés. Edgar Morin l'exprime ainsi dans Le Cinéma ou l'homme imaginaire: «Plus proche du cinéma est le rêve éveillé, lui-même à cheval entre la veille et le rêve» (p. 127). Plus loin, nous verrons en quoi la fiction interactive, bien que possédant certaines caractéristiques du cinéma, diffère d'attitude psychologique de par sa nature participative.

Edgar Morin compare l'état psychologique d'un cinéspectateur à l'état d'esprit du primitif et de l'enfant. Cet état d'esprit est bien semblable à ce que Carl Gustav Jung, à la suite de l'anthropologue Levy-Bruhl, appelle la participation mystique. Devant l'écran, dans la salle de cinéma, calés dans nos sièges, nous abandonnons nos méthodes intellectuelles de perception du monde pour retourner à une perception émotive des images, des sons et des sensations que nous acceptons comme réels, tout en n'ignorant rien de leur caractère tout à fait illusoire. Au cinéma nous savons que nous rêvons, nous nous laissons rêver voluptueusement, abdiquant notre volonté et notre activité au profit d'une torpeur pleine de sensations. À quoi bon protester contre tel ou tel choix de la réalisatrice ou de la scénariste. Le film est fait et le sort est jeté. Alea jacta est pourrait être la devise du cinéphile devant toute velléité de se rebeller contre le sort fait à ses héros. Durant un film nous n'avons aucun recours, nous sommes impuissants et même absents. Ce qui se passe devant nous ne fait pas partie de notre univers, c'est nous qui devenons l'univers du film. Un sentiment d'omnipotence narcissique nous envoûte si le film sait nous flatter avec talent.

Le spectateur de cinéma est passif, il ne peut rien faire pour influencer de quelque façon que ce soit ce qui se passe sur l'écran. Pour Edgar Morin, cette passivité est nécessaire au processus de projection-identification qui fonde le plaisir filmique. Sans cette passivité, il n'y aurait pas d'abaissement du niveau "perceptif» qui nous fasse accepter des incongruités telles que l'élasticité des échelles de plan, du temps, du point de vue, etc. Ce processus fait en sorte que tout ce qui se passe à l'écran devient pour nous une réalité intérieure et que nous objectivons cette réalité. L'écran occupe toute la place en nous et hors de nous et nous ressentons d'autant plus fortement les émotions suggérées par le film que nous sommes immobiles, inactifs, presque endormis, prêts à rêver. Cette dynamique a subi un premier choc avec l'avènement de la micro-informatique, elle a permis au spectacle de devenir participatif, d'abord dans les jeux vidéo puis dans toute la panoplie des jeux informatiques et des logiciels de 
créativité (traitement de texte, outil de dessin, de scénarisation, de composition musicale, etc.).

Alors le spectacle devient jeu, la passivité du spectateur fait place à l'activité excitante du joueur. Dans un jeu, non seulement la passivité disparaît, mais elle devient même nuisible à la bonne marche du jeu-spectacle informatique et interactif. Le fait de rester inactif devant une fiction interactive la rend inactive tandis qu'il donne au spectacle filmique ou télévisuel toute sa puissance. L'investissement énergétique qui était intériorisé dans le spectacle cinématographique doit s'actualiser dans des gestes qui se prolongent à l'intérieur de l'écran d'une fiction interactive. Mais si la passivité du spectateur n'est plus de mise dans un spectacle interactif, qu'en est-il des phénomènes psychologiques activés lors du spectacle filmique? Qu'en est-il de la participation mystique, de la projection-identification, de l'état onirique dans lequel nous sommes plongés? Le réalisme de l'image est-il affecté par l'activité du spectacteur? Y a t-il surcroît de sens critique gui nous fasse douter de l'illusion, rendant celle-ci caduque? Etant donné la nouveauté de cet art de l'interactif, nous ne pouvons qu'émettre des hypothèses sur sa nature psychologique.

À notre avis, la force expressive d'un art comme le cinéma ne tient pas tant à la nature passive du spectateur qu'à l'assurance de l'intégrité physique du spectateur. Nous sommes prêts à ressentir fortement un film dans la mesure où nous savons que rien ne peut nous arriver, et que notre sécurité est totale. Autrement, nous nous enfuirions de la salle au moindre danger apparent tout comme les premiers spectateurs du cinématographe au Grand Café de Paris, lorsqu'un train eut semblé paraître foncer sur eux. Ce sentiment de sécurité tient son efficacité de son ambiguïté. Nous savons qu'il n'y a aucun danger mais nous conservons un doute. Cette mise en confiance établie, nous sommes prêts à faire semblant que c'est dangereux. Car toute crainte réelle pour notre sécurité empêcherait l'illusion cinématographique. Â ce sujet, Edgar Morin écrit:

Dans tout spectacle et même s'il y a risque réel pour les acteurs, le public est en principe hors de danger, hors d'atteinte. Il est hors de portée du train qui arrive sur l'écran, lequel arrive présentement, mais dans un présent lui-même hors de portée du spectateur. Quoiqu'effrayé, celui-ci est tranquille. Le spectateur de cinématographe est non seulement pratiquement hors de l'action mais il sait que l'action, quoique réelle, se trouve actuellement hors de la vie pratique (p. 79).

Cependant, Morin ne donne pas à ce fait l'importance qu'il mérite, préférant concentrer ses efforts théoriques sur l'inhibition de l'action du spectateur et sa régression psychique: 
En situation régressive, infantilisé comme sous l'effet d'une névrose artificielle, le spectateur voit un monde livré aux forces qui lui échappent. C'est pourquoi, au spectacle, tout passe facilement du degré affectif au degré magique. C'est du reste dans la passivité-limite - le sommeil - que s'exagèrent les projections identifications qu'on appelle alors rêves (p. 81).

À partir du moment où il nous est permis de s'abandonner, nous sommes consentants à vivre par procuration tous les dangers physiques, moraux, ou sentimentaux que le cinéma nous montre à profusion. Il en est de même pour le sommeil: c'est une fois rassurés sur notre sécurité que nous pouvons dormir sur nos deux oreilles, tout danger réel nous forçant à ne dormir que d'un oeil. Tout danger réel provenant de l'illusion transforme celle-ci en réalité, et tout danger réel provenant hors de l'illusion nous empêche de nous y abandonner.

Arrêtons-nous un instant sur cette dernière phrase. Est-il vrai que tout danger réel provenant de l'illusion transforme celle-ci en réalité? Et si la fiction interactive avait comme caractéristique de transformer le danger illusoire par un danger similiréel? N'y a-t-il pas là surcroît de réalisme et d'émotions? Le spectacteur participe à une fiction interactive, il $\mathrm{y}$ joue un personnage qui peut être multiple, abstrait ou intégré dans la fiction. Ce personnage, au contraire du spectacteur lui-même qui le joue, court des dangers de toute sorte dans la fiction et, par conséquent, le spectacteur court lui-même des dangers à travers son personnage.

Bien qu'illusoires, les dangers deviennent réels par la médiation du personnage qu'interprète le spectacteur. Le spectacteur médiatise le personnage qui lui-même médiatise pour le spectacteur la réalité des dangers illusoires. Le spectacteur oublie sa condition humaine, revêt la peau d'un personnage et lui fait vivre, donc vit lui-même, les aléas de la fiction dans laquelle il est maintenant un élément qui contrôle, plus ou moins selon les œuvres, l'ensemble.

Bien qu'une communication interactive puisse s'en passer, la fiction de même nature fait appel, la plupart du temps, à ce personnage médiateur entre le spectacteur et la fiction. Quand nous parlons de ce personnage, il ne s'agit pas d'un personnage au sens strict, mais d'un faisceau de possibilités esthétiques de différentes formes que recouvre cette notion de personnage. Le contexte mis en place par le système peut faire appel à une communication directe entre la fiction et le public. L'intervention de celui-ci se fait alors au niveau formel. Le système sollicite un spectacteur placé au-dessus de la fiction qui doit juger des solutions idéales à un parcours personnalisé. Il y a alors séparation formelle entre 
le monde du spectacteur et celui de la fiction. Le spectacteur devant l'écran garde conscience de sa condition et son interaction se fait alors avec la globalité du système. Il n'oublie pas qui il est et ce qu'il fait, soit interagir avec une machine.

À travers un personnage, le spectacteur doit perdre cette conscience de se trouver devant un système interactif. Selon la qualité de la forme de l'interaction, le spectacteur doit confondre ses gestes envers le système avec les gestes du personnage à l'intérieur de la fiction. Le personnage assume les gestes du public et celui-ci se laisse convaincre qu'il n'est plus devant l'écran mais dans l'écran, avec ou dans cette forme visible qu'il manipule ou qui l'assiste.

Dans d'autres cas, le personnage (unique ou multiple) appartient à la fiction elle-même et sert au spectacteur à se retrouver au même niveau que tous les personnages de la fiction. Se retrouvant de plain-pied avec la fiction et les personnages qui l'habitent, il quitte le monde réel pour entrer dans celui d'un imaginaire réactif. Car si le propre de la communication entre le spectacteur et la fiction est d'être interactif, la fiction en ellemême pour le spectacteur est réactive, c'est-à-dire que ce qui devient apparent n'est pas le processus en tant que tel de l'interactivité mais plutôt la nature réactive de la fiction avec laquelle il joue. C'est à partir du moment où la fiction réagit qu'il lui est possible d'interagir avec elle.

Examinons maintenant les trois dynamiques de communication spectacteur-fiction que nous venons d'évoquer:

1-communication directe du spectacteur avec le système;

2-communication du spectacteur avec un personnage se faisant le médiateur du système;

3-communication du spectacteur avec un personnage qui le médiatise.

1- La première dynamique (communication directe du spectacteur avec le système) correspond, poussée à sa plus simple expression, à l'exploration manuelle d'un disque de fiction interactive. Le spectacteur contrôle la marche avant et arrière, les accélérés et les ralentis, la diffusion de chacune des deux pistes sonores, etc. Ou bien, plus couramment, les choix présentés par le système le sont de façon neutre: des textes ou des signes qui sont extérieurs à la fiction et se rapportent plutôt à la configuration du système comme, par exemple, des messages en modes vidéotex au cours d'une fiction dont l'esthétique n'a rien à voir avec celle du vidéotex, ou encore un plaquage d'instructions destinées directement au spectacteur dans une fiction qui semble ignorer sa présence. Toute intégration esthétique de la fiction et du proces- 
sus de communication avec le spectacteur tend à transformer ce dernier en personnage interne à la fiction. Cependant, il peut sûrement exister des cas où le processus de communication, malgré son intégration au contexte imaginaire présenté par la fiction, permet au spectacteur d'avoir un contact direct avec la fiction. Ce qui n'empêche pas le spectacteur d'être relégué dans son rôle de spectacteur interagissant avec un système diffusant un contexte imaginaire; il n'est pas soulagé de sa condition humaine en se transformant et en changeant sa dimension pour être englobé dans la fiction. La fiction reste pour lui un objet à manipuler plutôt qu'une situation à laquelle s'adapter.

Cependant ce type de dynamique, la communication directe avec la fiction, comporte aussi ce qu'on pourrait appeler la fiction à assembler. Tel un maître du jeu dans un jeu de rôle, qui prépare le terrain sur lequel les personnages des joueurs vont évoluer, semant les monstres, les pièges et les trésors, le spectacteur peut se faire le créateur d'une fiction dont les éléments sont réorganisés par lui dans le but de la faire actualiser par d'autres. Par exemple un disque laser accompagné d'un programme approprié peut présenter une fiction «en kit», un système imaginaire destiné à être organisé par un individu ou un groupe qui le présente alors à d'autres comme son œuvre personnelle. La réorganisation d'un tel genre de disque appelle une communication directe et active avec le système interactif présenté. Il est bien entendu qu'un tel type de fiction sollicite chez l'assembleur une créativité accrue. Il existe déjà ce qu'on appelle generic disc, c'est-à-dire un disque laser contenant toute une série d'images de différents sujets, en vrac, qu'un éducateur organise aux fins de ses besoins de démonstration ou pour souligner des aspects de son enseignement.

Quant aux fictions à assembler, pour être véritablement propices à la créativité, elles doivent permettre l'ajout d'éléments étrangers à son système que l'assembleur puisse intégrer et la possibilité pour ce dernier de modifier à sa guise les éléments déjà présents. À ce moment, une telle fiction devient un outil de créativité qui peut apporter bien des surprises quant à l'éducation du public et à l'évolution esthétique. Un tel outil devra permettre la production d'œuvres interactives mais aussi d'œuvres de fiction.

2- La deuxième dynamique (communication du spectacteur avec un personnage se faisant le médiateur du système) met en scène un personnage intermédiaire qui communique directement avec le spectacteur pour expliquer, sanctionner, féliciter, guider, etc. En fait, il incarne le système lui-même dans son rapport 
avec le contenu. Son articulation avec ce dernier doit être cohérente pour ne pas augmenter la distance entre le spectacteur et le contenu de l'œuvre. Ce personnage est soit à l'intérieur du contenu soit hors de ce contenu. Il n'y a pas identification directe du spectacteur à ce personnage, dans la mesure où il $\mathrm{y} a$ dialogue entre le personnage et le spectacteur et qu'on ne demande pas à ce dernier de se mettre à la place du premier.

3- Dans le dernier cas de dynamique de communication interactive (communication du spectacteur avec un personnage qui le médiatise) on peut parler de la différence entre une esthétique de personnage «subjectif» et de personnage «objectif». Ces deux qualificatifs sont utilisés dans leur sens habituel au cinéma, à savoir subjectif quand les scènes montrées suggèrent le point de vue d'un des personnages et objectif quand les scènes sont présentées en focalisation externe.

Pour le spectacteur, nous pouvons considérer que l'esthétique est subjective quand le personnage qu'il incarne n'est pas visiblement présent à l'écran mais que ce qui est présenté est ce qui lui arrive. Quant à l'esthétique objective, c'est à l'inverse quand le personnage que le spectacteur incarne est visible à l'écran et se fait manipuler par lui. Quelle est l'esthétique qui peut le mieux fasciner le spectacteur et l'amener à croire à ce qu'il vit? La question est lancée et ne pourra être répondue que lorsque des œuvres pourront être comparées dans cette optique. Pour l'instant, nous pouvons affirmer que les avis sont partagés.

Il ne faut pas négliger dans cette dynamique de communication entre le spectacteur et la fiction, la possibilité que le personnage puisse se présenter avec la propre image du spectacteur. Il suffit pour cela que l'image du spectacteur soit captée et intégrée à la fiction. Son image peut être numérisée ou tout simplement filmée, elle peut être modifiable ou non par le spectacteur, elle peut ou non accompagner une esthétique générale de plans subjectifs. Avec son image intégrée à la fiction, nous pouvons présumer que le processus de projection-identification tel que défini par Edgar Morin en est renforcé. Nous pouvons même considérer l'hypothèse d'une fusion, psychologiquement réelle, entre le personnage, la fiction et soi-même.

Revenons à la comparaison entre les effets psychologiques produits par le cinéma et ceux produits dans une fiction interactive utilisant comme support le disque laser. D'abord, l'inhibition motrice n'est plus l'état constant du spectateur; elle devient plutôt intermittente ou même absente selon le caractère de l'interactivité mise en scène. La concentration du spectateur devient nécessaire afin de repérer les moments où sa participation est requise et un effort de réflexion lui est demandé pour 
qu'il puisse adopter une tactique d'interaction. Quand et comment interagir sont des questions qui se posent d'abord aux concepteurs devant soigner le contexte d'interaction, puis ensuite au spectateur qui doit décider des gestes à poser pour que la fiction se déroule conformément à ses choix. Il y a donc, de la part du spectateur de fiction interactive, une attention qui n'est pas de la même nature que celle qui est demandée au spectateur de cinéma. Le premier n'étant pas exclu de la fiction, il dépasse l'état de «tout-percevant» (Christian Metz) du dernier pour s'approcher d'un état de «tout-agissant». La perception du spectateur de fiction interactive reste valorisée mais elle se met au service de son action. Nous ne pouvons plus affirmer du spectateur de fiction interactive (spectacteur) ce que Morin affirmait du spectateur de fiction linéaire:

Dans tout spectacle, avons-nous dit, le spectateur est hors de portée de l'action, privé de participations pratiques. Celles-ci sont, sinon totalement annihilées, du moins atrophiées et canalisées en symboles d'accompagnements (applaudissements) ou de refus (sifflets) et de toutes façons impuissantes à modifier le cours de la représentation. Le spectateur ne passe jamais à l'acte, tout au plus à des gestes ou des signes (p. 81).

Dans la fiction interactive, le spectateur passe à l'acte et le passage à l'acte constitue toute la richesse esthétique de cet art. Ce passage à l'acte qui est à l'origine du terme «interactif» fait partie d'un cycle communicationnel que nous nommons le cycle du contactile.

\section{Considérations sur le contactile}

De même que le mot spectacteur, le mot contactile est un motvalise. C'est la contraction des mots contact et tactile, c'est un contact tactile. C'est donc une action entraînant le sens du toucher. C'est un mot contenant à la fois la passivité et l'activité du toucher, on se fait toucher et on touche. Avant de développer cette notion de contactile, élaborons sur l'intrusion du sens du toucher dans un spectacle qui ne se voulait jusqualors qu'audiovisuel.

Contrairement à la vue et à l'ouie qui sont des sens de la distance, on ne peut toucher (et être touché) que ce qui est proche, contigu; c'est un sens intime. Et curieusement, les sens intimes comme le goût et le toucher ne font pas partie des sens que le cinéma a pu conquérir. D'abord, la vue bien sûr puis l'ouïe ont été intégrées au spectacle cinématographique. Puis il y eut et il continue à y avoir des tentatives d'odorifier le cinéma. Il y a même deux principales expérimentations de l'odeur à l'écran: 
l'odeur à gratter individuellement par chaque spectateur et l'odeur qui est soufflée dans la salle.

Il est curieux que l'une de ces expériences d'odorification du cinéma, baptisée Odorama, ait été une relative interactivisation du spectacle. Avec sa carte contenant la série d'odeurs à gratter au moment opportun du récit, le spectateur devient actif et peut même se permettre par humour ou curiosité de gratter au mauvais moment la mauvaise odeur, modifiant dans un sens non voulu par les producteurs du film le contenu esthétique du film. Cependant le procédé semble avoir tourné court et aucune nouvelle production en Odorama n'est annoncée.

Amener le sens du toucher dans le spectacle cinématographique ne peut se faire dans un cadre passif classique. Toucher c'est agir, et l'image cinématographique ne se laisse pas saisir. Alors si on ne peut toucher du doigt les objets de l'écran, on peut tout de même communiquer avec l'écran grâce au toucher. La révolution de la micro-informatique a permis cela. Une image vidéo reliée à un micro-ordinateur a toutes les qualités requises pour devenir une image «digitale». Manipuler une manette, un clavier ou un écran tactile est une opération «digitale». La main étant active, la condition de passivité devient caduque.

La notion de sécurité aussi peut changer dans le cadre d'une communication interactive. Rien n'interdit à la communication digitale de se faire dans les deux sens. Si un effleurement du doigt modèle la fiction, le système, insatisfait de cet effleurement, peut de diverses façons agresser physiquement le spectacteur.

Un choc électrique, un jet d'eau, un bruit strident peuvent faire partie de la panoplie des réponses du système aux contacts du spectacteur. Nous voulons dire par là qu'une fiction interactive est tactile lors de la communication entre le spectacteur et le système, tout comme la communication entre le système et le spectacteur peut se faire de façon tactile. Cependant la signification de chacune des directions de la «tactilité» de la communication interactive est différente.

Quand nous qualifions de «digital» le processus de communication mis en branle lors de l'interprétation d'une fiction interactive, c'est par généralisation. Bien que la façon habituelle, actuelle, de communication entre le spectacteur et la fiction interactive passe par le bout des doigts (clavier, écran tactile) ou par la manipulation d'un objet (manette de jeu, boule, souris, crayon lumineux, etc.), c'est le corps au complet ou par portion qui est invité à participer à la communication. Le développement des interfaces indique bien que tout mouvement ou pulsation du corps peut être récupéré en tant qu'émetteur et porte-parole du 
spectacteur. Le mouvement des yeux, les ondes électroencéphalographiques, les pulsations cardiaques, le déplacement du corps dans un espace, les mouvements de la tête ou des jambes..., le corps même du spectacteur, et quelquefois même les mouvements inconscients ou incontrôlables de son corps deviennent alors le véritable lieu de la fiction, le générateur et le récepteur de la fiction, l'acteur et la scène. Le centre de la fiction se déplace alors de l'écran, comme au cinéma, à la télévision, à la vidéo, vers le spectacteur et son corps signifiant. Ce déplacement de polarité dans le processus de diffusion d'une fiction audiovisuelle médiatisée est une transformation ontologique du concept de fiction.

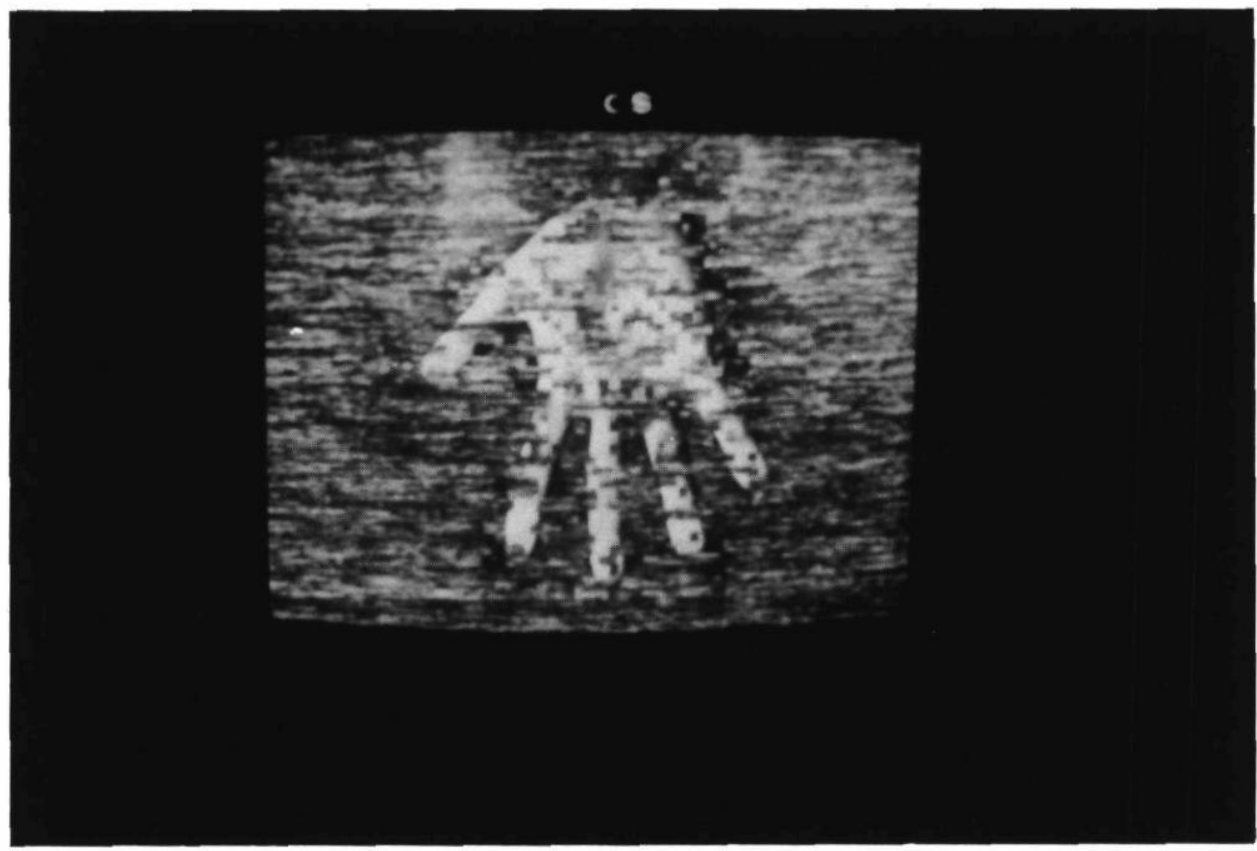

Premier contact de Réjean Dumouchel, Louis Fortier, Diane Grenier, Marie Grenier, Wilfrid Lamoureux. Les Productions Louise Grenier (1987) Photo: Réjean Dumouchel

De la «digitalisation» à la «tactilisation», voire l'impact physiologique de la communication directe vers le corps des données de la fiction, comme, par exemple, dans le fait de relayer vers le spectacteur la douleur ressentie par le héros, se continue l'évolution classique de la technologie des arts de diffusion en série, à savoir l'accroissement de la saturation des sens dans un 
but de réalisme accru. C'est la version tactile de l'Odorama. Ce qui n'empêche pas cette dynamique de diffusion tactile de la fiction d'ouvrir de nouvelles voies esthétiques et formelles assez attirantes pour les artistes sensuels ou... sadiques. Imaginons la richesse d'une scène offrant un double contrepoint sonore et tactile à une image tridimensionnelle. Voilà peut-être, énoncée ici, la principale caractéristique des chefs-d'œuvre de l'avenir.

De même la fiction tactile remet-elle en cause notre hypothèse de la nécessité de l'immunité du spectateur comme condition de sa participation émotive à la fiction. Dans certains de ces cas, lorsque la communication tactile du système vers le spectateur peut être source de danger physique, il y a concentration des sens du spectateur face à la fiction, afin de s'éviter dans la mesure du possible les désagréments physiques que peuvent lui causer les réactions du système à ce qui est considéré par lui comme des mauvais choix lors de la communication digitale. Ce qui logiquement devrait activer notre émotivité face aux péripéties de la fiction puisqu'elles concernent directement notre confort physique.

Bien que largement hypothétiques, en ce sens que de telles fictions tactiles n'existent pas encore à notre connaissance, ces considérations sur les conséquences de la «tactilisation» du spectacle audiovisuel ne veulent circonscrire que ce qui est faisable grâce à l'utilisation du disque vidéo interactif comme support de la fiction. Puisqu'un lien physique est nécessaire entre le système et le spectacteur pour que la communication interactive se fasse, ce même lien peut véhiculer de l'information tactile autant qu'audiovisuelle. Cette information peut provenir du spectacteur ou lui être destinée. Et cette dimension supplémentaire de la fiction ne constituerait qu'une étape de plus dans la quête du spectacle total à laquelle le développement technologique actuel nous invite.

Quoiqu'il en soit de cet aspect de la «digitalisation» de la fiction à cause de la nécessité physique de l'interaction entre le spectacteur et le système interactif, c'est de la participation corporelle du spectacteur à la fiction que nous voulons parler. Pour cela, il nous faut revenir au concept de contactile.

Le contactile est le concept qui recouvre le cycle de communication tel que caractérisé par la fiction interactive. Au sens premier, le contactile est le geste que doit poser le spectacteur pour signifier au système sa décision. C'est le doigt qui touche une zone active de l'écran tactile, ce sont les doigts qui tapent et envoient au système une directive, c'est la nouvelle direction que la main donne à la manette, c'est le bouton que le doigt presse... 
Le contactile est, en fait, le noeud de la fiction interactive. À un autre niveau, le contactile est le témoignage actif de la présence du public et le relais de sa volonté. C'est aussi la principale différence entre une fiction interactive et une fiction linéaire. Cette dernière ne possède pas de contactile car elle est indépendante des réactions du public tandis que la fiction interactive s'appuie sur ces réactions, en est dépendante.

Le geste signifiant du public envers le système s'inscrit, nous l'avons écrit plus haut, dans un cycle de communication que nous avons nommé, par extension, de ce même terme de contactile. Pour qu'il $\mathrm{y}$ ait geste du public envers le système qui opère la fiction interactive, il faut qu'il y ait un contexte établi par les concepteurs et les producteurs de la fiction qui attire ce geste, l'appelle et le justifie. Le contactile fait partie de l'esthétique d'une œuvre interactive et en constitue l'expression privilégiée puisqu'il intègre le public dans le processus de représentation de la fiction. Nous avons donc, pour l'instant, un cycle de communication d'une œuvre qui place d'une part le contexte mis en place pour favoriser l'interaction et d'autre part le spectacteur disposé ou non à interagir avec le contexte présenté.

Le lien qui unit le spectacteur au contexte est le contactile, qui, par l'action que le spectacteur doit poser pour répondre au contexte, intègre le public dans la représentation de l'œuvre ou encore, vu d'un autre angle, transcende l'œuvre, en lui faisant accéder à la réalité psycho-physique du public qui lui donne les caractéristiques organiques qui lui manquaient.

Une œuvre est un objet, mais grâce au contactile, elle devient sujet. La fiction perd son statut d'histoire pour acquérir celui d'aventure vécue. Vécue non par procuration mais par performance. Par le contactile, l'œuvre se désobjectivise pour se subjectiviser quand elle se réalise. Il n'y a plus devant soi un objet à contempler, mais un objet à manipuler. Par cette manipulation, une œuvre réussie convainc le spectacteur qu'il ne fait pas face à un objet indépendant mais à un moment de sa vie qui transforme ses mouvements corporels en tranches de vie fictive.

Nous avons ici trois éléments qui composent le cycle du contactile, c'est-à-dire le contexte, le spectacteur et entre les deux, le geste médiateur appelé contactile. Pour que le cycle soit complet, il faut ajouter un quatrième élément qui est la réaction du système au contactile qui amène une modification au contexte, invitant à un nouveau contactile. Si le contactile n'apporte aucune modification visible ou invisible au contexte, il est inutile et il n'y a pas d'interaction. 
Voici donc comment se déroule le cycle du contactile. D'abord le système opérant la fiction présente un contexte invitant le public à l'interaction, puis le public accepte le contexte en faisant contact avec celui-ci par le biais du contactile. Ce dernier est évalué par le système qui réagit en modifiant le contexte de façon à encourager le public à produire un nouveau contactile. S'établit alors une communication véritablement interactive en ce sens que le public, par le contact, modifie le contexte et que ce dernier appelle un nouveau contact pour qu'une nouvelle modification, dépendant de la forme du contact, s'effectue.

Le cycle du contactile circonscrit donc le processus de communication qui s'effectue lors de la représentation d'une fiction interactive. Il indique aussi que dans le cadre d'une œuvre de ce genre, le public est inséparable de la diffusion de l'œuvre et en fait donc partie. Une œuvre interactive n'existe pas par ellemême, elle n'existe qu'en tant que performance ponctuelle par un ou des spectacteurs d'un certain nombre des virtualités présentes dans le système opérant l'œuvre.

Bien que la notion de contactile recouvre toute une dynamique physio-psychologique qui rend compte de l'implication corporelle du spectacteur dans le déroulement de la fiction et à cause de cela même, il y a une autre dynamique, plus complète, à l'origine et à l'aboutissement du contactile, celle de l'auteur face à son œuvre. À l'évidence, les stratégies de formalisation de son œuvre sont différentes et spécifiques à la communication interactive. D'abord la technologie y tient une grande place parce qu'elle doit servir à la fois de médiateur de la fiction vers un spectateur, mais aussi de médiateur du spectacteur vers la fiction et surtout elle doit servir de lien structurel entre les attentes et les réponses du système et du spectacteur.

La technologie doit alors remplir trois rôles: diffuseur de la fiction, récepteur du public (interface), «structureur» de la communication. Ce dernier rôle est en fait un rôle d'interlocuteur véritable du public, qui ne communique avec la fiction que par le biais de l'interprétation structurante de son message.

\section{Montage ou montages?}

Un disque laser, comme un film, a été monté selon l'art du raccord, du rythme et de l'expressivité, inventé par la cinématographie puis transposé à la vidéo et au vidéodisque. À l'inverse des deux premiers, le vidéodisque n'a pas à tenir compte de la linéarité de son montage mais plutôt de l'espace de son montage. Le montage d'un vidéodisque interactif est à deux dimensions. Il doit tenir compte de la place qu'occupe une image sur la surface 
du disque et de la proximité relative des prochaines images probables. Le montage cinéma et vidéo est un montage de certitude, un montage à une dimension. Une fois le montage terminé, il est certain qu'une séquence sera suivie lors de sa projection de la séquence qui la suit matériellement. Sur un disque vidéo, il n'y rien de certain dès qu'un noeud contactile intervient, il faut alors pour optimiser l'espace qu'occupe la totalité des images, distribuer celles-ci selon des règles de probabilités de raccords. Le principal but d'un tel montage est souvent de raccourcir au maximum le temps que prend la tête de lecture pour aller chercher le segment approprié. Cela devient un montage probabiliste.

Par calcul ou par intuition, les segments sont placés à la file pour qu'une fois gravés à la surface du disque, la majorité des choix offerts par un contactile occupe un temps de réponse minimum. L'ennemi à combattre est le temps mort durant lequel l'image et le son disparaissent pendant que la tête de lecture laser se déplace vers la piste appropriée au contactile effectif et s'y accroche pour la diffuser.

Quand on regarde la disposition des images sur un disque laser, on ne peut que constater qu'il y existe trois types de montage, trois moments où le montage change de nature. Ces trois temps du montage d'une fiction interactive lui sont caractéristiques et apportent un surcroît de travail à la conception pour un surcroît de plaisir à la réception. Il y a d'abord, sur le disque lui-même, le montage linéaire dont nous avons vu qu'il doit s'organiser d'une façon différente que dans la fiction linéaire. Il y a, sous forme de structure logicielle, le montage virtuel qui prévoit si possible toutes les virtualités inhérentes à la fiction gravée sur le disque. Il y a finalement le montage actuel qui réalise un certain faisceau des possibilités du montage virtuel par le biais de la performance du spectacteur. Examinons maintenant chacun de ces montages pour en observer quelques aspects.

Le montage linéaire est ce qui se retrouve sur le disque, c'està-dire une succession d'images gravées sur sa surface et lues par le rayon laser. Il est la réplique du montage effectué sur ruban magnétique. Si on met le disque en marche en dehors de son asservissement logiciel, nous allons voir le montage linéaire d'un bout à l'autre, de la première à la dernière image.

Ce montage doit sa cohérence non pas à la succession des différentes séquences qui le composent mais à l'organisation de ces séquences sur le disque, de façon à optimiser l'actualisation du montage virtuel. Son organisation dépend souvent de la proximité relative des séquences entre elles, de leur probabilité d'être 
choisies lorsque le bras de lecture se trouve dans telle position sur la surface du disque. Habituellement, les scènes fréquemment appelées doivent être au milieu du montage linéaire afin qu'elles ne soient jamais à une distance maximale d'une autre séquence susceptible d'être appelée. De la même façon les images ou séquences rarement appelées doivent être situées au début ou à la fin du montage linéaire qui correspondent respectivement au centre axial et à la périphérie du disque. Il peut aussi être utile de répéter une image ou une courte séquence qui revient constamment afin qu'elle soit toujours à proximité d'une scène probable. Quant au contenu du montage linéaire, il a bénéficié des mêmes étapes de montage que n'importe quel vidéo professionnel. En fait le montage linéaire ne se suffisant pas à luimême dans une fiction interactive, il doit obéir à l'esthétique du montage virtuel.

Le montage virtuel correspond à la structure logicielle de la fiction interactive. Ce montage, qui n'en est pas un au sens physique du terme est toutefois le plus délicat des trois; celui qui règle le reste. Un montage linéaire désorganisé peut toutefois être réchappé par un montage virtuel bien conçu, mais un mauvais montage virtuel torpille les qualités d'une fiction interactive.

Le montage virtuel, c'est l'ensemble de toutes les possibilités de raccords permises par la structure de la fiction. Il relève d'une exploration méthodique de ces virtualités au moment de la conception de la fiction et de sa programmation. D'un même disque au montage linéaire fixe, il peut être tiré un nombre infini de montages virtuels variés.

En fait, nous pouvons considérer ce montage comme le montage des contactiles. C'est donc un montage qui prend en considération le spectacteur et ses réactions au contexte. Présenté sous forme numérique sur disquette ou disque dur, ce montage peut inclure toute la partie infographique et fonctionner sur deux ou plusieurs plans simultanés quand il est possible de synchroniser les signaux vidéo issus du vidéodisque et ceux issus de la disquette. Mais le montage virtuel peut aussi être présent sur le disque optique, attendant de se décharger temporairement dans le système informatique pour devenir effectif.

Le troisième moment du montage est ce que nous appelons le montage actualisé, c'est-à-dire ce qui advient lors de la représentation de la fiction. Le montage actualisé, c'est le montage virtuel que le spectacteur réalise en partie lors de sa performance. C'est la personnalisation du montage virtuel. Chaque contactile appelle une des possibilités du montage virtuel à se diffuser et à être vécue par le spectacteur et ce dernier actualise le montage virtuel en lui associant sa performance personnelle. 
Bien évidemment, aucun montage actualisé ne peut épuiser la totalité du montage virtuel à moins que ce dernier soit singulièrement pauvre ou que la performance du spectacteur soit exhaustive, ce qui est peu probable. Mais la plupart du temps le montage actualisé dépend du lieu, du moment et de la personne qui interagit. Il s'agit donc de l'inscription de la fiction dans la réalité objective et psychologique comme il s'agit aussi réciproquement de l'intrusion de la réalité dans la fiction. Cette réalité est celle du spectacteur qui, dans le circuit de la communication d'une fiction interactive, est le seul élément vivant, donc le seul témoin du réel.

Par le contactile qui actualise le montage virtuel, le spectacteur insuffle la vie à la fiction interactive. Si le concepteur est le créateur de la fiction, le spectacteur lui donne véritablement naissance.

Alors que la fiction interactive possède ces trois niveaux de montage, au cinéma il n'y a qu'un niveau, qui confond le montage linéaire avec le montage actualisé, mais ne connaît pas le montage virtuel. En réalité seul le montage linéaire existe au cinéma et les montages virtuel et actualisé sont des nouveautés qu'il nous faudra apprendre à apprivoiser. Il faudra apprendre à produire des fictions probabilistes dans lesquelles le public joue aux dés. Les raccords ne sont plus certains comme au cinéma lors de la projection, mais doivent tenir compte des probabilités d'actualisation par le spectacteur.

L'abandon des certitudes, malgré son aspect insécurisant et peut-être à cause de cela même, constitue le premier sacrifice que le ou les concepteurs doivent faire pour réussir une fiction interactive. Il n'y a plus de recettes, il n'y a que des aventures esthétiques qui peuvent (et doivent) être détournées par le spectacteur. Une fiction interactive doit accroître la liberté du spectacteur, lui donner la chance d'être créateur à son tour.

Nous avons tenté, tout au long de ce texte, de rechercher dans la technologie du disque laser ce qui en fait un support nouveau pour un art inouï. Car, croyons-nous, il y a ici dans ce support, somme toute technologique, tous les éléments pour qu'une esthétique dont la sensibilité nous est inconnue puisse émerger. Il est certain que «l'apparition de nouvelles techniques de production des images n'entraîne pas nécessairement ou automatiquement une conception renouvelée de l'image» (Payant, p. 234), mais dans le cas du disque laser nous pouvons affirmer que tel est bien le cas.

Par ses caractéristiques formelles, par son caractère interactif, le disque laser nous invite à réévaluer notre conception de 
l'image pour que celle-ci puisse profiter pleinement du potentiel créatif du disque laser. L'art de la fiction interactive ou plus simplement de l'image et du son interactifs demande de la part de ceux et celles qui l'exploitent une ouverture d'esprit spécifique, une audace formelle particulière.

Cet art est le fruit de l'évolution technologique et de l'évolution esthétique. La technologie recherchait un support pour que le cinéma puisse se diffuser dans les foyers, tandis que l'art se cherchait un support qui puisse contenir des «œuvres en mouvement». Le disque laser n'a pas atteint les objectifs commerciaux que ses inventeurs visaient; il a permis la naissance non pas du cinéma chez soi mais du cinéma interactif, qui est venu presque «envers et contre tous». Aujourd'hui cependant l'idée de la fiction interactive entre dans les moeurs et dans les circuits commerciaux. Les romans «dont vous êtes le héros», les jeux d'aventure informatiques, la télévision interactive, les soirées mystère où il faut résoudre en groupe l'énigme d'un crime fictif, témoignent tous de ce mouvement qui donne au public une place, petite ou grande, sur la scène ou dans l'écran.

Les masses-médias semblent vouloir perdre un peu de leur hégémonie sur le public au profit des récits «personnalisés». Nous pouvons ressentir une certaine similitude avec la naissance du cinéma. Si nous nous fions à ce que les inventeurs du septième art recherchaient, nous nous approchons peut-être de cette conception originelle.

Leur imagination identifie l'idée cinématographique à une représentation totale et intégrale de la réalité, elle envisage d'emblée la restitution d'une illusion parfaite du monde extérieur avec le son, la couleur et le relief (Bazin, p. 22).

Ce que ces gens cherchaient, c'était le spectacle total, la représentation globale. Toutes les recherches de la technologie cinématographique visaient à accroître le réalisme de la représentation et à magnifier les sensations que le cinéma peut provoquer. $\mathrm{Ce}$ qui fait dire à André Bazin que «tous les perfectionnements que s'adjoint le cinéma ne peuvent donc paradoxalement que le rapprocher de ses origines» (p. 23).

C'est pourquoi nous croyons qu'un perfectionnement aussi transcendant (puisqu'il donne accès au spectateur à l'écran) que l'interactivité ramène le cinéma au moment de son origine. La fiction interactive, ce n'est déjà plus du cinéma mais ça lui ressemble, en ce sens qu'elle constitue le plus puissant médium de représentation de la fiction qui soit et qu'elle attend des artistes qu'ils en façonnent le langage expressif. 
Comme avec Méliès, à la naissance du cinématographe, qui trouva là la scène privilégiée de ses explorations formelles, l'art de l'interactif demande à être malmené, subverti. Un illusionniste de la trempe de Méliès a fait du médium de la réalité quotidienne des frères Lumière la plus formidable machine à illusions de son époque. À notre tour, il faut voir jusqu'à quel point le disque laser et la fiction interactive peuvent nous émouvoir, nous émerveiller et nous leurrer. Et surtout, jusqu'où un spectacteur par ses contactiles peut se libérer de sa passivité pour entrer de plain-pied dans une fiction qui devient la sienne.

Université de Montréal

\section{OUVRAGES CITÉS}

Bazin, André. Qu'est-ce que le cinéma? Paris: Cerf, 1985.

Corneau, Guy. «Narcisse au cinéma». «De la représentation à l'énigme?». Cahiers de psychologie jungienne 43 (4e trimestre 1984).

Éco, Umberto L'Euvre ouverte. Paris: Seuil, 1979.

Metz, Christian. «Le Film de fiction et son spectateur». Communications 23 (1975).

Metz, Christian. Le Signifiant imaginaire. Paris: U.G.E., 1977.

Morin, Edgar. Le Cinéma ou l'homme imaginaire. Essai d'anthropologie. Genève: Gonthier, 1965.

Payant, René. «Le Postmodernisme selon le cinéma». Le Cinéma aujourd'hui: Films, théories, nouvelles approches (dir. Michel Larouche). Montréal: Guernica, 1988. 